Investitionen der Energieversorger in erneuerbare Energien

\title{
Von grünen Riesen und grauen Schleiern
}

\begin{abstract}
Seit einiger Zeit nehmen die vier großen Energieversorger in Deutschland viel Geld für erneuerbare Energien in die Hand und vermarkten dies sehr effektiv. Wie viel jedoch in Wirklichkeit investiert wird und ob sie damit die politischen Ziele erreichen, wurde in einer neuen Studie untersucht. Von Bernd Hirschl
\end{abstract}

\begin{abstract}
A s RWE Mitte dieses Jahres mit der Figur eines grünen Riesen Kinowerbung machte, war ein neuerlicher Höhepunkt in der Vermarktung der Energiekonzerne als umweltfreundliche Unternehmen erreicht. Bereits zuvor gab es zahlreiche andere Werbemaßnahmen in gleicher Richtung, auch von den anderen drei größten in Deutschland aktiven Energieversorgern EON, Vattenfall und EnBW. Darin wurden unter anderem Meereskraftwerke beworben, die es noch nicht am Markt gibt oder eine erneuerbare Energien-Welt dargestellt, die sehr wenig mit dem realen Geschäft der Konzerne zu tun hat.

Über die vorhandenen und geplanten Erzeugungskapazitäten im Bereich der erneuerbaren Energien (EE) stellen die Konzerne aber keine vollständigen Daten zur Verfügung.
\end{abstract}

\section{Viel Kohle und viel Wind...}

Es zeigt sich, dass alle vier Konzerne von einem sehr niedrigen Ausgangswert starten, wenn man das Jahr 2007, in dem sie ihre Bemühungen in diesem Bereich erst richtig begonnen haben, zum Ausgangspunkt nimmt. Während 2007 der EE-Anteil in Deutschland bereits bei 14,2 Prozent lag, erreichten die Konzerne bei den neu errichteten Technologien, also ohne Berücksichtigung von alten Wasserkraftanlagen, Werte von 0,1 Prozent (EnBW), 0,4 Prozent (RWE), 1,1 Prozent (Vattenfall) und 1,4 Prozent (EON). Nimmt man die Wasserkraft hinzu, stei- gen die Anteile insbesondere bei EON auf 7,2 Prozent und bei EnBW auf 11,4 Prozent. Konzernweit sehen die EE-Anteile ohne Wasserkraft ähnlich aus. Berücksichtigt man auch hier die Wasserkraft, dann steigt der Wert insbesondere beim schwedischen Versorger Vattenfall aufgrund der dort vorhandenen vielen Anlagen auf etwa 21 Prozent. Damit ist Vattenfall als einziger Konzern bereits nah an der gegenwärtigen Zielmarge der Europäischen Union für 2010.

Bei der Analyse zeigte sich zunächst, dass alle Konzerne es bisher vermieden haben, Planungen und Ziele in einer zu den politischen Zielvorgaben vergleichbaren Form anzugeben. Ganz im Gegenteil wurden eine Reihe von Verschleierungen vorgenommen. Hierzu gehört, dass der Anteilswert auf der Basis der vergleichsweise höheren Anlagenkapazitäten anstelle der Stromerzeugung angegeben wird, oder dass zu den Erneuerbaren fälschlicherweise auch der gesamte Abfall oder Pumpspeicherkraftwerke gezählt werden. Diesbezüglich sind deutlich genauere und standardisierte Angaben einzufordern.

Alle Konzerne peilen ein enormes Wachstum an. EON und Vattenfall wollen beispielsweise ihre Kapazitäten bis 2030 annähernd verdoppeln. Den größten Anteil dieses Wachstums sollen fossile Kraftwerke und Atomstrom übernehmen, auch Letzterer soll über 50 Prozent wachsen. Bei der Kohle sind nur leichte Verschiebungen zu Gas geplant, und in welchem Umfang die Kohlenstoffdioxid-Abscheidung und
-Speicherung eine Rolle spielen soll, wird überwiegend nicht festgelegt.

Auch die Erneuerbaren sollen stark anwachsen, allerdings erreicht bis 2020 nur Vattenfall aufgrund der hohen Wasserkraftanteile den vorgesehenen Zielwert von 30 Prozent im Strombereich, EnBW folgt mit 21 Prozent. EON und RWE bleiben mit 15 beziehungsweise 12 Prozent sogar noch unter dem allgemeinen Zielwert der Europäischen Union von 20 Prozent. Der mit deutlichem Abstand größte Anteil am EE-Ausbau der Konzerne soll dabei auf die Windenergie entfallen, insbesondere im Offshore-Bereich. Stärker dezentrale Technologien werden kaum eine Rolle spielen.

\section{... passen nicht zusammen}

So begrüßenswert das Engagement der großen Vier im Bereich Offshore und anderer EE-Großprojekte durchaus ist, umso bedenklicher ist der gleichzeitige geplante Ausbau von größeren fossilen und auch nuklearen Kraftwerkskapazitäten. Wie viele Experten mittlerweile betonen, passen das alte Grundlastkonzept und ein hoher Anteil fluktuierender erneuerbarer Energien nicht zusammen (SRU 2009). Eine intelligentere und flexiblere Systemintegration ist notwendig und schafft gleichzeitig die Voraussetzung für mehr Energieeffizienz. An Transparenz und Schlüssigkeit mangelt es also nicht nur den Daten, sondern auch den gesamten längerfristigen Energiekonzepten der Konzerne.

\section{Literatur}

Hirschl, Bernd: Investitionen der vier großen Energiekonzerne in Erneuerbare Energien; http://www.ioew.de (16.9.2009)

SRU (Sachverständigenrat für Umweltfragen): Weichenstellungen für eine nachhaltige Stromversorgung; Mai 2009. http:// www.umweltrat.de (16.9.2009)

I AUTOR + KONTAKT

Dr. Bernd Hirschl ist Leiter des Forschungsfelds Nachhaltige Energiewirtschaft und Klimaschutz am IÖW.

IÖW, Potsdamer Str. 105, 10785 Berlin. Tel.: +4930 884594-0,

E-Mail: bernd.hirschl@ioew.de; Internet: http://www.ioew.de 
(c) 20I0 Authors; licensee IÖW and oekom verlag. This is an article distributed under the terms of the Creative Commons Attribution Non-Commercial No Derivates License (http://creativecommons.org/licenses/by-nc-nd/3.o/), which permits unrestricted use, distribution, and reproduction in any medium, provided the original work is properly cited. 